\title{
3.6 Два подхода к прогнозированию временных рядов
}

Построение модели системы производится по наблюдаемым величинам. В медицине, экологии, социологии динамику объекта исследования отслеживают по временным реализациям - временным рядам. Как правило, при анализе временных рядов используются методы, дающие количественный прогноз (точечный или интервальный). Для временных рядов, у которых гипотеза о существовании тренда не подтверждается, такие методы не продуктивны.

Применение методов теории нелинейных динамических систем к анализу временных рядов основано на предположении, что имеющийся ряд описывает поведение изучаемой системы, и это единственная доступная информация о ней. По известной теореме Такенса [220] для адекватного описания динамической системы в целом достаточно единственного временного ряда.

Анализ временных рядов методами теории нелинейных динамических систем приобретает все более широкое распространение. По терминологии этой теории процесс, описываемый временным рядом, содержит в себе детерминированный хаос, или, проще говоря, является хаотическим. С точки зрения линейных методов анализа - это стохастические процессы. Нелинейный анализ показывает, что с одной стороны, эти процессы нельзя причислить к детерминированным, с другой стороны, абсолютно случайными они также не являются.

Сегодня теория хаоса остается одним из самых распространенных способов прогнозирования и исследования устойчивости состояний динамических систем. Целью анализа устойчивости системы является выявление всех ее стационарных состояний. Если хотя бы одно из стационарных состояний по каким-либо причинам оказывается угрожающим или нежелательным, то его наличие дает возможность выработать превентивные меры, уменьшающие вероятность перехода системы в это состояние.

Одной из часто встречающихся форм потери устойчивости является хаотизация состояния системы [220]. Механизмы перехода системы к такому 
состоянию изучены недостаточно. Однако знание того, что такое состояние возможно, требует поиска новых методов исследования систем. Инструментами исследования в теории хаоса являются аттракторы и фракталы. Два наиболее распространенных критерия хаотичности динамических систем - это показатель Ляпунова и фрактальная размерность.

В рамках второго подхода авторы предлагают рассматривать временной ряд $\left\{x_{n}\right\}$ как последовательность регистрируемых сигналов, которые порождаются некоторым случайным процессом. Измерения $x_{n}(n=\overline{1, N})$, как правило, подвержены шуму, который затрудняет описание процесса, не позволяет получить достоверную информацию об источнике сигналов. Для таких стохастических процессов не существует точных математических моделей, что значительно затрудняет анализ и прогнозирование соответствующих временных рядов. Универсальным аппаратом описания подобного рода стохастических процессов является математический аппарат скрытых моделей Маркова (CMM). СММ близки к хорошо известным цепям Маркова. Модели на базе цепей Маркова относятся к структурным моделям, в которых зависимость будущего от прошлого задается в виде некоторой структуры и правил перехода по ней.

Отличие этих двух теорий состоит в том, что СММ рассматривают изучаемый случайный процесс как смену неизвестных фактических состояний некоторой системы $\Omega$, а значения $x_{n}(n=\overline{1, N})$ временного ряда как ее выходные значения.

\subsection{1. Предпрогнозный анализ временных рядов методами} фрактального анализа и фазовых траекторий

В работе [221] обосновываются причины малой информативности статистических показателей как следствие наличия фрактальных свойств в поведении временных рядов, эмпирическая функция распределения которых не согласуется с нормальным распределением. Поэтому для обнаружения общей 
тенденции поведения временных рядов предлагается на этапе предпрогнозного анализа использовать методы теории хаоса, которые дают возможность провести качественный анализ исследуемого временного ряда. По отношению к динамике социально-природных систем и процессов теория хаоса не только объясняет бифуркационные явления (большие падения или большие выбросы), но и утверждает, что их невозможно предсказать, поскольку природа не есть ряд повторяющихся закономерностей, и характеризуется локальной случайностью и глобальным порядком. По этой причине многие аналитики обоснованно предположили, что распознать в хаотическом движении новые закономерности им поможет фрактальная природа временных рядов [222-225].

К формальным характеристикам хаотических процессов в теории нелинейных динамических систем относятся, в первую очередь, фазовое пространство и аттрактор. Одной из особенностей хаотического поведения системы является неустойчивость траекторий, принадлежащих аттрактору. Количественной мерой этой неустойчивости являются характеристические показатели Ляпунова. Поскольку критерием хаотической динамики является наличие положительного старшего показателя Ляпунова, то естественный интерес представляет возможность его оценки на основании обработки заданного временного ряда [225]. В случае критерия хаоса достаточно вычислить только наибольший показатель Ляпунова.

Нелинейные динамические системы обычно имеют фрактальные аттракторы, то есть неустойчивые фазовые траектории систем с течением времени стремятся стать фракталами [221]. Важным моментом во фрактальном подходе является влияние предыстории случайного процесса на поведение системы сегодня.

Одним из самых востребованных направлений фрактального анализа является изучение динамики во времени такой характеристики, как фрактальная размерность. Этот показатель характеризует повторяемость статистических характеристик естественных временных рядов с изменением масштаба. Фрактальная размерность, введенная Хаусдорфом как $D$ - размерность, является 
основной характеристикой фрактальных структур [225, 226].

Имеется несколько методов определения фрактальной размерности для временного ряда, рассматриваемого как совокупность наблюдаемых параметров изучаемой динамической системы во времени. Во-первых, это классический способ клеточного покрытия графического изображения временного ряда, при котором фрактальная размерность определяется точно так же, как и для геометрических фракталов. Второй способ для исследования фрактальных временных рядов был предложен Бенуа Мандельбротом. Он базируется на исследованиях английского ученого Херста и носит название $R / S$ метода.

Для большинства реальных временных рядов аналитическое нахождение фрактальной размерности невозможно. Поэтому величину $D$ определяют численно, например, через показатель Херста $H$. Влияние настоящего на будущее при анализе временного ряда может быть выражено корреляционным соотношением $C=2^{2 H-1}-1$ [227].

Если в качестве аппроксимации временных рядов рассматривать совокупность плоских геометрических фигур (клеток) с общим геометрическим параметром $\delta$, то по определению Хаусдорфа $D$-размерность определяется из закона $S(\delta) \sim \delta^{2-D}$ при $\delta \rightarrow 0$, где $S(\delta)$ - площадь всей совокупности клеток с масштабом разбиения $\delta$.

Одним из показателей стабильности временного ряда является индекс фрактальности $\mu$ [229-231]. Преимущество этого индекса перед другими фрактальными показателями состоит, в частности, в том, что для его определения с приемлемой точностью достаточно данных на два порядка меньше, чем, например, для определения значения показателя Херста $H$. Это дает возможность проводить локальный фрактальный анализ временных рядов на основе свойств функции $\mu(t)$.

Один из распространенных способов визуальной оценки временного ряда опирается на процедуру восстановления фазовой траектории. Возможности такого визуального анализа фазового портрета весьма ограничены. Однако для 
идентификации сложных непериодических временных реализаций анализ фазовых портретов дает порой больше информации, чем спектральный анализ данных. Преимуществом этого способа является также то, что он применяется независимо от того, построена или нет модель объекта исследования. Поскольку на практике далеко не всегда удается получить подходящую модель, то реальным способом анализа устойчивости системы остается безмодельный способ анализа поведения фазовых траекторий.

В работе авторов [228] проведен анализ устойчивости значения показателя заболеваемости различными кожными патологиями в Украине по его временным реализациям с помощью анализа фазовых траекторий и качественных методов фрактального анализа.

Определение фрактальной размерности для временного ряда было проведено классическим способом клеточного покрытия графического изображения временного ряда.

На рис. 1 изображен анализируемый временной ряд $\left\{x\left(t_{i}\right)\right\}_{i=1}^{N} \quad$ и построенное для него минимальное покрытие.

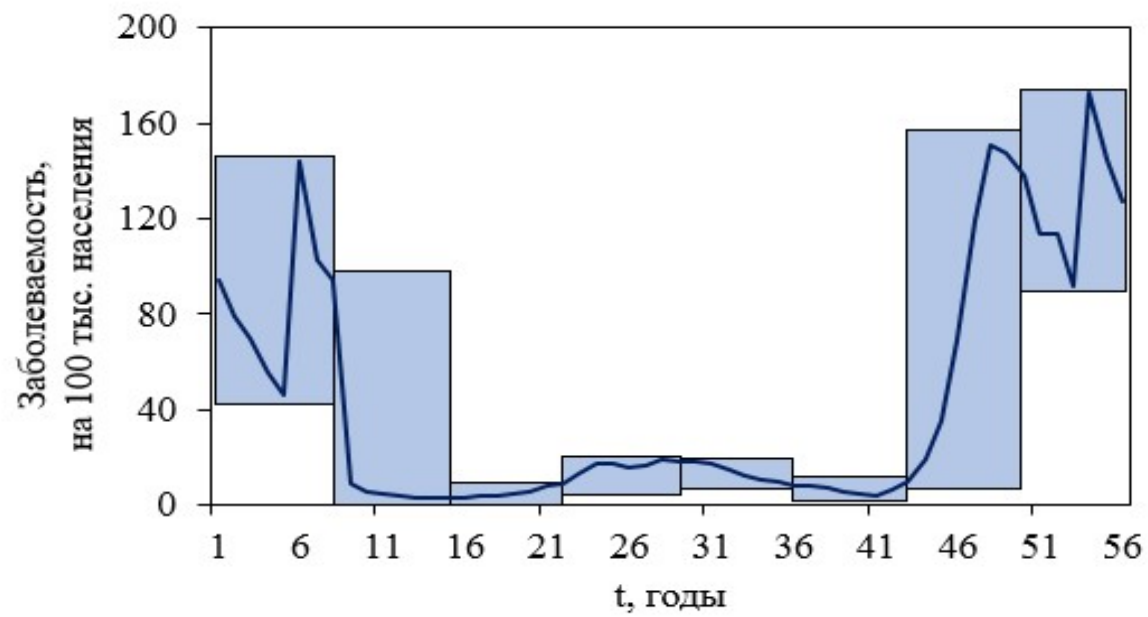

Рисунок 1. Минимальное клеточное покрытие для временного ряда, характеризующего заболеваемость некоторыми кожными патологиями в Украине с 1958 года.

При уровне надежности $\alpha=0,90$ индекс фрактальности исследуемого ряда 
оказался равным $\mu=0,66 \pm 0,073$.

Оценка старшего показателя Ляпунова $\lambda\left(x_{1}\right)$ осуществлялась авторами по методу сравнения точек эволюции фазовой траектории. В соответствии с этим методом оценку $\lambda\left(x_{1}\right)$ для траектории $x_{i}=x\left(t_{i}\right), i=\overline{1, N}$, дискретного временного ряда $x_{t+1}=f\left(x_{t}\right)$ авторы проводили по формуле

$$
\lambda\left(x_{1}\right)=\lim _{N \rightarrow \infty} \frac{1}{N} \sum_{i=1}^{N} \ln \left|f^{\prime}\left(x_{i}\right)\right|,
$$

соответствующей определению $\lambda\left(x_{1}\right)$ при условии, что предел справа существует. Проведенные расчеты показали, что для заданного временного ряда $\lambda\left(x_{1}\right) \approx 1,18$, то есть $\lambda\left(x_{1}\right)>0$, а следовательно, траектория хаотическая.

Фазовый портрет позволяет выявить особенности поведения системы, важные с точки зрения устойчивости. Для поиска аттрактора в случае двух (трех) факторов строится фазовое пространство и анализируется положение фазовых точек. При их равномерном распределении гипотеза о существовании аттрактора не подтверждается.

На рис. 2 представлены результаты построения фазовых портретов для временного ряда, представленного на рис. 1. Величина временной задержки $\tau$ составляет 1 год.

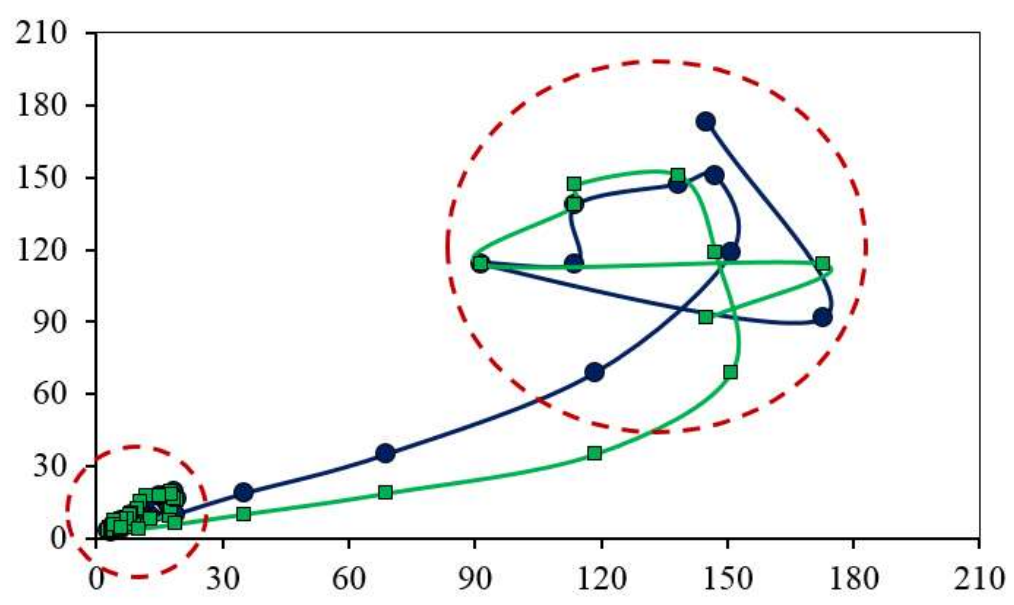

Рисунок 2. Фазовые портреты временного ряда.

Как видно из рис. 2, существует две области сгущения фазовых точек. Их 
можно рассматривать как расщепленный аттрактор.

Другими словами, имеет место бифуркация аттрактора. Обычно это связывают с появлением у системы таких изменений состояния, которые могут быть интерпретированы как скачкообразные или близкие к ним. При анализе медицинских данных бифуркация аттрактора влечет за собой с большой вероятностью внезапное кризисное изменение состояния системы.

3.6.2. Прогнозирование временных рядов методом скрытых марковских моделей

Наиболее широкое применение СММ нашли при распознавании речи и анализе изображений [232-234], а также при моделировании финансовых рядов с целью построения решающих правил торговли на финансовых рынках [235, 236].

В настоящих исследованиях аппарат СММ рассматривается применительно к анализу и прогнозированию медико-социальных временных рядов. Медико-социальные ряды отличаются от финансовых не только количеством измерений, но и частотой измерений в единицу времени, что делает медико-социальные ряды существенно инерционными.

Теория СММ не является новой. Первые публикации по этой теме появились еще в 60 - 70-х годах прошлого века. Но тогда широкого развития эта теория не получила ввиду сложности вычислительной части исследований. Поэтому практические аспекты применения метода СММ к прогнозированию временных рядов проработаны недостаточно.

В настоящее время развивается новый подход к моделированию временных рядов, учитывающий структуру корреляции данных. Это динамические байесовские сети [237,238], которые применительно к задаче прогнозирования временных рядов выступают в форме СММ.

Учитывая сказанное выше, актуальной задачей является разработка основ моделирования с целью прогнозирования медико-социальных временных рядов с использованием математического аппарата СMM.

Рассмотрим некоторую динамическую систему $\Omega$, которая в 
произвольный момент времени $t$ может находиться в одном из возможных состояний $S_{1}, S_{2}, \ldots, S_{N}$. В дискретные моменты времени система меняет состояние (возможно, переходя в то же состояние). В этом случае соответствующий случайный процесс представляет собой последовательность событий вида $\left\{S(t)=S_{i}\right\} \quad(i=\overline{1, N}, t=1,2, \ldots)$ (в момент времени $t$ система находится в состоянии $S_{i}$ ). Переходы из состояния в состояние происходят под действием случайных факторов. Наиболее важной характеристикой этой цепи событий являются вероятности состояний системы: $p_{i}(t)=P\left\{S(t)=S_{i}\right\}$. Распределение этих вероятностей - одномерный закон распределения случайного процесса с дискретными состояниями и дискретным временем. Такой процесс называется Марковской цепью.

Полное вероятностное описание системы $\Omega$ предполагает задание текущего состояния $S(t)$ и всех предыдущих. В случае Марковского случайного процесса вероятность каждого из состояний $S(t+1)$ системы в будущем зависит лишь от ее состояния $S(t)$ в настоящем, и не зависит от того, когда и как она пришла в это состояние. Другими словами, вероятностное описание такой системы требует знания текущего и предыдущего состояний системы, то есть сводится к виду:

$$
P\left\{S(t+1)=S_{j} \mid S(t)=S_{i}, S(t-1)=S_{k}, \ldots\right\}=P\left\{S(t+1)=S_{j} \mid S(t)=S_{i}\right\} .
$$

Далее рассмотрим процессы, при которых правая часть равенства (1) не зависит от $t$, то есть не зависят от $t$ вероятности перехода (переходные вероятности) из состояния $S_{i}$ в состояние $S_{j}$ :

$$
a_{i j}=P\left\{S(t+1)=S_{j} \mid S(t)=S_{i}\right\},
$$

при этом $a_{i j} \geq 0$ и $\sum_{j=1}^{M} a_{i j}=1$. В этом случае цепи Маркова называются однородными. Принципиальным в наших исследованиях является наличие 
стационарного режима в цепи Маркова, то есть такого ее состояния, при котором система $\Omega$ продолжает переходить из состояния $S_{i}$ в состояние $S_{j}$, но вероятности этих состояний не зависят от $t: p_{i}(t)=p_{i}$. Для существования стационарного режима в цепи Маркова ее однородность является необходимым условием.

В случае стационарного режима в однородной Марковской цепи достаточно просто решаются задачи прогнозирования возможных состояний системы $\Omega$ в таких формулировках:

- какова вероятность того, что в следующие $k$ дней последовательность смены состояний будет иметь заранее заданный вид?

- какова вероятность того, что система останется в состоянии $S_{i} k$ дней?

- каково наиболее вероятное число повторений одного и того же состояния в течении $k$ дней и др.

В случае временного ряда $\left\{x_{n}\right\}, n=\overline{1, N}$, зададим $\left\{x(t)=S_{i}\right\}(i, t=\overline{1, N})$ как состояние $S_{i}$ системы $\Omega$ в момент времени $t$. При этом равенство (2) для переходных вероятностей примет вид:

$$
a_{i j}=P\left\{x(t+1)=S_{j} \mid x(t)=S_{i}\right\} .
$$

В основе построения СММ временного ряда лежит основной принцип теории цепей Маркова, но при условии, что фактические (скрытые) состояния $Q_{i}(i=\overline{1, K})$ системы $\Omega$ и их количество не известны, а имеются лишь выходные данные $x_{n}(n=\overline{1, N})$. Скрытая Марковская цепь также представляет собой некоторую последовательность состояний $Q_{i}(i=\overline{1, K})$ в дискретные моменты времени. Переход из состояния $Q_{i}$ в состояние $Q_{j}$ происходит также случайно с вероятностью $a_{i j}(3)$.

В случае временного ряда $\left\{x_{n}\right\}, n=\overline{1, N}$, построение СММ с целью 
прогноза его состояний требует задания таких элементов модели:

1. $K$ - количество состояний системы в модели; $Q=\left\{Q_{1}, Q_{2}, \ldots, Q_{K}\right\}-$ множество состояний; $q_{t}$ - состояние системы в момент времени $t, t=\overline{1, N}$.

2. $M$ - количество символов алфавита наблюдаемой последовательности состояний; $V=\left\{V_{1}, V_{2}, \ldots, V_{M}\right\}$ - алфавит возможных символов.

3. $A=\left\|a_{i j}\right\|$ - матрица вероятностей перехода, где $a_{i j}=P\left\{q_{t+1}=Q_{j} \mid q_{t}=Q_{i}\right\}$ $(i, j=\overline{1, K}, t=\overline{1, N})$.

4. $B=\left\|b_{i}(m)\right\|$ - матрица вероятностей появления символов $V_{m}(m=\overline{1, M})$ алфавита $V$ в состоянии $Q_{i}(i=\overline{1, K})$, то есть $b_{i}(m)=P\left\{V_{m} \mid q_{t}=Q_{i}\right\}-$ вероятность того, что система, находящаяся в состоянии $Q_{i}$ в момент времени $t$, выдаст символ $V_{m}$.

5. $\pi=\left\{\pi_{1}, \pi_{2}, \ldots, \pi_{K}\right\}-$ распределение вероятностей начального состояния, где $\pi_{i}=P\left\{q_{1}=Q_{i}\right\}$, то есть $\pi_{i}-$ вероятность того, что в начальный момент времени $(t=1)$ система находилась в состоянии $Q_{i}(i=\overline{1, K})$.

Совокупность введенных в пп. 1-5 величин $(N, K, A, B, \pi)$ определяет CMM.

В работе авторов [239] построение СММ выполнялось для временного ряда, впервые рассмотренного авторами в работе [228].

По результатам расширенного теста Дики - Фуллера (ADF-теста) с константой без тренда исходный временной ряд не является стационарным. В случае временного ряда первых разностей $\Delta x_{t}=x_{t+1}-x_{t}, t=\overline{1, N-1}$, оказалось, что можно сделать вывод о стационарности ряда первых разностей. Поэтому построение СММ проводилось для временного ряда первых разностей

$$
\left\{\Delta x_{n}\right\}, n=\overline{1, N-1} \text {. }
$$


На рис. 3 приведены графики исходного временного ряда и ряда (4) его первых разностей.

Для определения скрытых $Q_{i}(i=\overline{1, K})$ состояний модели Маркова временного ряда (4) первых разностей наблюдаемой последовательности состояний был проведен кластерный анализ значений этого ряда.

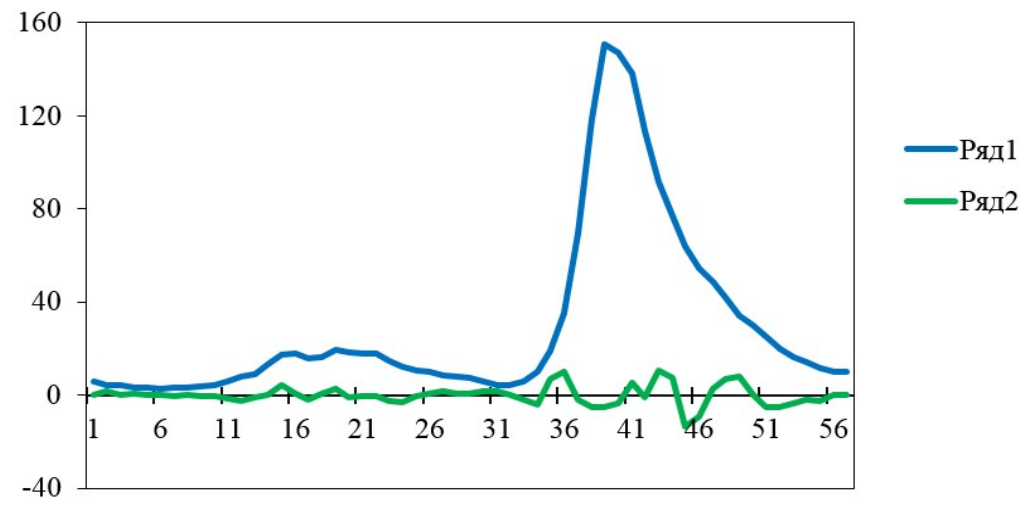

Рисунок 3. Ряд 1 - исходный ряд $\left\{x_{n}\right\}, n=\overline{1, N}$; ряд 2 - ряд первых разностей

$$
\left\{\Delta x_{n}\right\}, n=\overline{1, N-1} \text {. }
$$

Оптимальное число кластеров, а, значит, и фактических (скрытых) состояний $Q_{i}(i=\overline{1, K})$ в модели Маркова временного ряда (4), оказалось равным 3. Результаты кластерного анализа приведены на рис. 4.

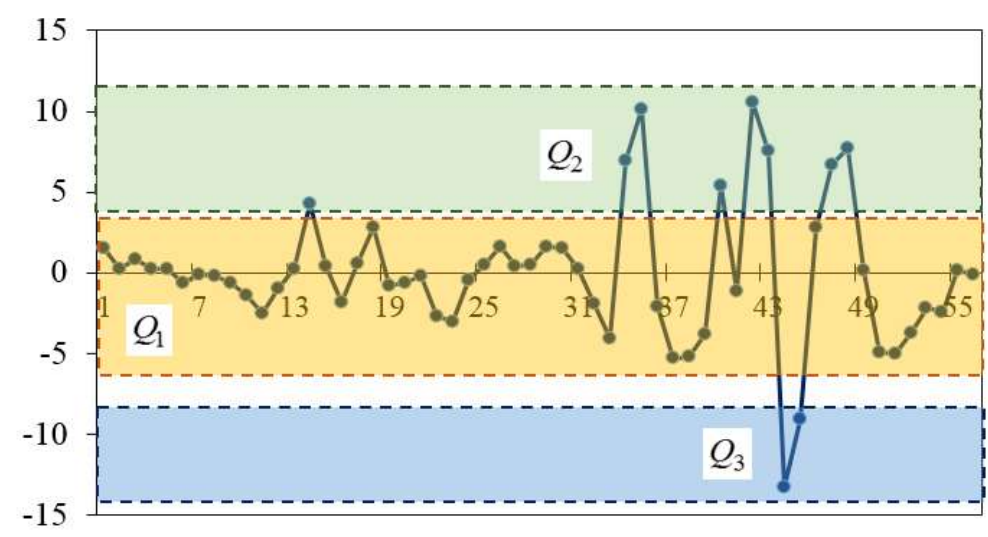

Рисунок 4. Результаты кластерного анализа временного ряда первых разностей.

Анализ графика временного ряда первых разностей, построенного по 
результатам кластерного анализа (представлен на рис. 3), дал возможность остановиться на построении $3 \times 3$ - модели СММ, где количество символов алфавита $V$ также равно 3 , то есть $V=\left\{V_{1}, V_{2}, V_{3}\right\}$. Для создания алфавита $V$ в случае трех скрытых состояний системы воспользовались следующей традиционной терминологией: $V_{1}-« ф л э т », V_{2}-\left\langle\right.$ «рост», $V_{3}-$ «падение».

Для определения начального распределения связей между состояниями $Q_{i}(i=\overline{1,3})$ модели цепи Маркова была принята гипотеза о зависимости возможностей переходов между состояниями модели от соответствующих расстояний между ними. Используя данные об евклидовых расстояниях между центрами кластеров, определили возможности переходов между состояниями и их направления:

Шаг 1. Устанавливается возможность перехода для пары состояний, если их центры находятся на минимальном расстоянии или превышают его не более чем на заданный процент. На практике обычно выбирают 50 \%-й порог.

Шаг 2. Направление переходов между состояниями определяется в соответствии с близостью к начальному состоянию $Q_{1}:$ переход $Q_{i} \rightarrow Q_{j}$ возможен, если число переходов $Q_{1} \rightarrow Q_{j}$ больше числа переходов $Q_{1} \rightarrow Q_{i}$. Если направление не определяется однозначно, то допускаются оба варианта направления: $Q_{i} \square Q_{j}$.

Следует заметить, что в построенной по таким правилам модели цепи Маркова допускаются косвенные и статистически незначимые связи. Поэтому следует провести уточнение схемы, например, с использованием статистики Пирсона.

Наличие начального распределения связей дает возможность для скрытых состояний $Q_{i}(i=\overline{1,3})$ составить матрицу переходных вероятностей $A=\left\|a_{i j}\right\|$, где $a_{i j}=P\left\{q_{t+1}=Q_{j} \mid q_{t}=Q_{i}\right\},(t=\overline{1, N}, i, j=\overline{1,3}) ; q_{t}-$ состояние системы в момент 
времени $t$. Матрица $A=\left\|a_{i j}\right\|$ и граф модели Маркова представлены на рис. 5

Распределение вероятностей начального состояния $-\pi=\left\{\pi_{1}, \pi_{2}, \pi_{3}\right\}-$ в случае ряда (4) имеет вид: $\pi=\{1,0,0\}$. Построение алфавита $V$ проведено по результатам кластерного анализа, который дал возможность сформировать матрицу $B=\left\|b_{i}(m)\right\|$ вероятностей появления символов $V_{m}(m=\overline{1,3})$ алфавита $V$ в состоянии $Q_{i}(i=\overline{1,3})$.

$$
A=\left(\begin{array}{ccc}
\frac{40}{45} & \frac{5}{45} & 0 \\
\frac{4}{8} & \frac{3}{8} & \frac{1}{8} \\
\frac{1}{2} & 0 & \frac{1}{2}
\end{array}\right)
$$

$a$

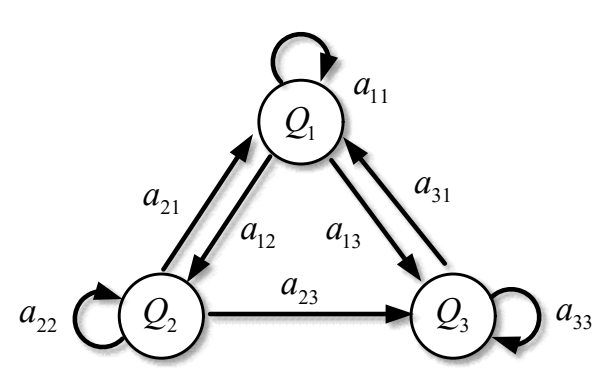

$\sigma$

Рисунок 5. $a$-матрица переходных вероятностей; 6 - граф скрытой модели Маркова.

Построенная СММ для ряда (4) первых разностей опирается на два предположения, обусловленные стационарностью этого ряда. Во-первых, текущее состояние модели $q_{t}$ зависит лишь от предыдущего состояния $q_{t-1}$. И, во-вторых, вероятность появления некоторого наблюдения $o(t+1)$ в следующий момент времени зависит только от текущего состояния системы $q_{t}$ и не зависит от предыдущих состояний. Из рис. 5 видно, что $q_{t}=q_{56}=Q_{1}$. Тогда наиболее вероятным состоянием $o(t+1)$ системы будет такой прогнозный результат: $a_{11}=P\left\{q_{57}=Q_{1} \mid q_{56}=Q_{1}\right\}=\frac{8}{9}$. Вероятность наблюдения, например, такой цепочки $\quad$ состояний: $O: Q_{1} \rightarrow Q_{1} \rightarrow Q_{2} \quad$ вычисляется $\quad$ таким $\quad$ образом: $p(O)=P\left\{q_{59}=Q_{2} \mid q_{58}=Q_{1}, q_{57}=Q_{1}\right\}=\frac{8}{9} \cdot \frac{8}{9} \cdot \frac{1}{9}=\frac{64}{729} \approx 0,088$. 